C. Iwasaki and Y. Morimoto

Nagoya Math. J.

Vol. 101 (1986), 111-130

\title{
PRECISE PROPAGATION OF SINGULARITIES FOR A HYPERBOLIC SYSTEM WITH CHARACTERISTICS OF VARIABLE MULTIPLICITY
}

\author{
CHISATO IWASAKI AND YOSHINORI MORIMOTO
}

\section{Introduction}

In this paper we consider the Cauchy problem for a hyperbolic system with characteristics of variable multiplicity and construct a certain solution whose wave front set propagates precisely along the so-called "broken null bicharacteristic flow", in other words, along the admissible trajectory if we use the terminology of [6].

Let $L$ be a hyperbolic system of the form

$$
\left[\begin{array}{cc}
D_{t}+\lambda_{1}\left(t, x, D_{x}\right) & 0 \\
0 & \ddots \\
D_{t}+\lambda_{l}\left(t, x, D_{x}\right)
\end{array}\right]+B(t, x) \quad \text { on } \quad R_{t}^{1} \times R_{x}^{n},
$$

where $\lambda_{j}(t, x, \xi)$ are linear in $\xi$, that is,

$$
\lambda_{j}(t, x, \xi)=\sum_{k=1}^{n} a_{j, k}(t, x) \xi_{k}, \quad j \in\{1, \cdots, \ell\} .
$$

Here $a_{j, k}(t, x) \in C^{\infty}$ are real-valued and polynomials of first order with respect to $x$;

$$
a_{j, k}(t, x)=\sum_{m=1}^{n} C_{j, k}^{m}(t) x_{m}+C_{j, k}^{0}(t)
$$

We assume that the term of order zero $B(t, x)=\left(b_{j, k}(t, x)\right)$ satisfies

$$
b_{j, k} \neq 0 \quad \text { if } \quad j \neq k .
$$

We consider the Cauchy problem

$$
L U=0, \quad U(0, x)=G, \quad G \in \mathscr{E}^{\prime}
$$

and show the precise propagation of the wave front set of a solution along

Received October 8, 1984. 
the admissible trajectory, by assuming some hypotheses (See Theorem 1 in $\S 1)$. Hypotheses of Theorem 1 are fairly complicated, but they are satisfied for some cases discussed in [6] and [7], for instance, we can apply Theorem 1 to the proof of Theorem 2 of [7] (see also Theorem 2 of [6] and its 87).

In the last section, as another application of Theorem 1 we give an example of hyperbolic system $L$ on $R_{t}^{1} \times R_{x}^{1}$, for which there exists an initial value $G$ such that

$$
\text { sing supp } G=\{0\}, \quad \text { sing } \operatorname{supp} U(T)=[-1,1],
$$

where $U$ is the solution of (C.P) and $T$ is a fixed positive. For a hyperbolic equation of second order, Ichinose-Kumano-go [5] gave a result similar to (0.5) by using the work of Taniguchi-Tozaki [12], though we do not know whether the Cauchy problem for their equation is well-posed until $t=T$ (see Theorem 3.5 of [5]).

In the next paper [11], the proof of Theorem 1 will be applied to show the precise propagation of wave front sets in Gevrey classes along "generalized null bicharacteristic flow" defined by Kumano-go-Taniguchi [9] and Wakabayashi [13] [14].

\section{$\S 1$. Notations and main result}

We say that a curve $\{t, x(t), \xi(t)\} \subset R_{t}^{1} \times T^{*} R_{x}^{n}$ is the bicharacteristic curve with respect to $\lambda_{j}$ through $(s, y, \eta)$ if it satisfies equations

$$
\left\{\begin{array}{l}
d x / d t=d_{\xi} \lambda_{j}(t, x, \xi), \quad d \xi / d t=-d_{x} \lambda_{j}(t, x, \xi) \\
\left.(x, \xi)\right|_{t=s}=(y, \eta) .
\end{array}\right.
$$

For the abbreviation we write bic. curve w.r.t. $\lambda_{j}$ in what follows. We denote by $\chi_{j}(t, s)$ the transformation from the cotangent space $T^{*} R_{x}^{n}$ to itself such that $\left(t, \chi_{j}(t, s)(y, \eta)\right)$ is bic. curve w.r.t. $\lambda_{j}$ through $(s, y, \eta)$. Since $\lambda_{j}$ are linear in $\xi$ and $a_{j, k}$ polynomials of the first order with respect to $x$, there exist a matrix $M_{j}(t, s)$ and a vector $d_{j}(t, s)$ such that

$$
\chi_{j}(t, s)(y, \eta)=\left(M_{j}(t, s) y+d_{j}(t, s),{ }^{t} M_{j}(t, s)^{-1} \eta\right),
$$

where ${ }^{t} M^{-1}$ denotes the inverse of the transposed matrix of $M$.

For an integer $\nu \geqq 0$ let $J_{\nu}=\left(j_{1}, j_{2}, \cdots, j_{q}, \cdots, j_{\nu+1}\right)$ be a $(\nu+1)$-repeated permutation of elements $\{1,2, \cdots, \ell\}$, that is, $j_{q} \in\{1, \cdots, \ell\}$ for $1 \leqq q \leqq \nu+1$. Let $\Pi_{\nu}$ be a set of all $J_{\nu}$ and let $\Pi_{\nu}^{0}$ be a set of all $J_{\nu}$ satisfying $j_{q} \neq j_{q+1}$ for $1 \leqq q \leqq \nu$. 
For a fixed $t_{0}>0$ let $\Delta_{\nu}^{0}$ denote an open set of $R^{\nu}(\nu \geqq 1)$ as follows; $\Delta_{\nu}^{0}=\left\{\tilde{t}_{\nu}=\left(t_{1}, \cdots, t_{\nu}\right) ; t_{0}>t_{1}>t_{2}>\cdots>t_{\nu}>0\right\} . \quad$ A continuous curve $\left\{(t, x(t), \xi(t)) ; t \in\left[0, t_{0}\right]\right\}$ is called a trajectory of step $\nu$, issuing from $\rho \in$ $T^{*} R^{n} \backslash 0$ if for some $J_{\nu} \in \Pi_{\nu}^{0}$ and some $\tilde{t}_{\nu} \in \Delta_{\nu}^{0}$ it is bic. curve w.r.t. $\lambda_{j_{q}}$ when $t \in\left[t_{q}, t_{q-1}\right]\left(q=1, \cdots, \nu+1, t_{\nu+1}=0\right)$ and $(x(0), \xi(0))=\rho$. We denote this trajectory by $C\left(J_{\nu}, \tilde{t}_{\nu}, \rho\right)$. A point

$$
\chi_{j_{1}}\left(t_{0}, t_{1}\right) \chi_{j_{2}}\left(t_{1}, t_{2}\right) \cdots \chi_{j_{\nu+1}}\left(t_{\nu}, 0\right) \rho
$$

is called the end point (at $t=t_{0}$ ) of the trajectory. It follows from (1.2) that the end point is equal to

$$
\left(M_{J_{\nu}}\left(\tilde{t}_{\nu}\right) y+d_{J_{\nu}}\left(\tilde{t}_{\nu}\right),{ }^{t} M_{J_{\nu}}\left(\tilde{t}_{\nu}\right)^{-1} \eta\right), \quad \rho=(y, \eta)
$$

where a matrix $M_{J_{\nu}}$ and a vector $d_{J_{\nu}}$ are defined by, respectively,

$$
\begin{aligned}
M_{J_{\nu}}\left(\tilde{t}_{\nu}\right)= & M_{j_{1}}\left(t_{0}, t_{1}\right) M_{j_{2}}\left(t_{1}, t_{2}\right) \cdots M_{j_{\nu+1}}\left(t_{\nu}, 0\right) \\
d_{J_{\nu}}\left(\tilde{t}_{\nu}\right)= & d_{j_{1}}\left(t_{0}, t_{1}\right)+M_{j_{1}}\left(t_{0}, t_{1}\right) d_{j_{2}}\left(t_{1}, t_{2}\right)+\cdots \\
& +M_{j_{1}}\left(t_{0}, t_{1}\right) \cdots M_{j_{\nu}}\left(t_{\nu-1}, t_{\nu}\right) d_{j_{\nu+1}}\left(t_{\nu}, 0\right) .
\end{aligned}
$$

Definition 1.1. We say that a trajectory $C\left(J_{\nu}, \tilde{t}_{\nu}, \rho\right)$ is $\varepsilon$-admissible for a $\varepsilon \geqq 0$ if we have

$$
\left|\left(\lambda_{j_{q}}-\lambda_{j_{q+1}}\right)\left(t_{q}, x^{q}, \xi^{q}\right)\right| \leqq \varepsilon\left|\xi^{q}\right| \quad \text { for any } q \in\{1, \cdots, \nu\},
$$

where $\left(x^{q}, \xi^{q}\right)=\chi_{j_{q+1}}\left(t_{q}, t_{q+1}\right) \cdots \chi_{j_{\nu+1}}\left(t_{\nu}, 0\right) \rho$. We say only admissible in place of 0 -admissible.

We remark that a bic. curve is always an adm. traj. of step 0. For the brevity we often write adm. traj. instead of admissible trajectory.

For $J_{\nu}=\left(j_{1}, \cdots, j_{\nu+1}\right) \in \Pi_{\nu}^{0}$ and $J_{\mu}^{\prime}=\left(j_{1}^{\prime}, \cdots, j_{\mu+1}^{\prime}\right) \in \Pi_{\mu}^{0}$ we write $J_{\nu} \supset$ $J_{\mu}^{\prime}$ if there exists a subset $\left\{q_{1}^{0}, q_{2}^{0}, \cdots, q_{r}^{0}, \cdots, q_{\mu+1}^{0} ; q_{r}^{0}<q_{r+1}^{0}\right\}$ of $\{1, \cdots, \nu+1\}$ such that $j_{q_{r}^{0}}=j_{r}^{\prime}$ for $r=1, \cdots, \mu+1$. Obviously, if $J_{\nu} \supset J_{\mu}^{\prime}$ then $\nu \geqq \mu$.

Definition 1.2. For a small $\varepsilon_{0}>0$ and a fixed trajectory $C_{\mu}=$ $C\left(J_{\mu}^{\prime}, \tilde{t}_{\mu}^{\prime}, \rho\right)$ we say that a trajectory $C\left(J_{\nu}, \tilde{t}_{\nu}, \rho\right)$ is contained in $\varepsilon_{0}$-neighborhood of $C_{\mu}$ if and only if $J_{\nu} \supset J_{\mu}^{\prime}$ and the following conditions are satisfied: Suppose that

$$
J_{\nu}=\left(j_{1}, \cdots, j_{q}, \cdots, j_{\nu+1}\right), \quad \tilde{t}_{\nu}=\left(t_{1}, \cdots, t_{q}, \cdots, t_{\nu}\right) .
$$

When $\mu \geqq 1$, suppose that

$$
J_{\mu}^{\prime}=\left(j_{1}^{\prime}, \cdots, j_{r}^{\prime}, \cdots, j_{\mu+1}^{\prime}\right), \quad \tilde{t}_{\mu}^{\prime}=\left(t_{1}^{\prime}, \cdots, t_{r}^{\prime}, \cdots, t_{u}^{\prime}\right) .
$$


Then there exists a subset $\left\{q_{1}, \cdots, q_{\mu}\right\}$ of $\{1, \cdots, \nu\}$ such that

$$
\left|t_{q_{r}}-t_{r}^{\prime}\right|<\varepsilon_{0}, \quad r=1, \cdots, \mu .
$$

And furthermore we have

$$
\begin{aligned}
& \left|t_{q}-t_{q-1}\right|<\varepsilon_{0} \quad \text { for any } q \in\{1, \cdots, \nu+1\} \text { satisfying } \\
& q_{r} \geqq q>q-1 \geqq q_{r-1} \text { and } j_{q} \neq j_{r}^{\prime}, \quad r=1, \cdots, \mu+1,
\end{aligned}
$$

where $q_{0}=0, q_{\mu+1}=\nu+1$ and $t_{\nu+1}=0$.

When $\mu=0$, suppose that $J_{\mu}^{\prime}=(j)$. Then $J_{\nu}=(j)$ if $\nu=0$. If $\nu \geqq 1$ we have (1.7) for any $1 \leqq q \leqq \nu+1$ satisfying $j_{q} \neq j$.

ThEOREM 1. We assume that

$(\mathrm{H}-1)\left\{\begin{array}{l}\text { There exists an admissible trajectory } C_{\mu}=C\left(J_{\mu}^{\prime}, \tilde{t}_{\mu}^{\prime} \cdot \rho_{0}\right) \text { of step } \mu \text { issuing } \\ \text { from } \rho_{0}=\left(y^{0}, \eta^{0}\right) \in T^{*} R^{n} \backslash 0 \text { whose end point } \\ \text { is } \delta_{0}=\left(x^{0}, \xi^{0}\right)\left(=\left(M_{J_{\mu}^{\prime}}\left(\tilde{t}_{\mu}^{\prime}\right) y^{0}+d_{J_{\mu}^{\prime}}\left(\tilde{t}_{\mu}^{\prime}\right),{ }^{t} M_{J_{\mu}^{\prime}}\left(\tilde{t}_{\mu}^{\prime}\right)^{-1} \eta^{0}\right)\right) .\end{array}\right.$ (H-2) $\left\{\begin{array}{l}\text { If } \mu \geqq 1 \text { then it follows that } \\ \operatorname{det}\left(\partial_{t_{p}} \partial_{t_{q}}\left(\xi^{0} \cdot\left(M_{J_{\mu}^{\prime}}\left(\tilde{t}_{\mu}^{\prime}\right) y^{0}+d_{J_{\mu}^{\prime}}\left(\tilde{t}_{\mu}^{\prime}\right)\right)\right)\right) \neq 0, \quad(p, q=1, \cdots, \mu) .\end{array}\right.$

$(\mathrm{H}-3)\left\{\begin{array}{c}\text { Let } V_{\varepsilon} \text { be an open } \varepsilon \text {-conic neighborhood of } \xi^{0}, \\ \text { There exist an open neighborhood } V_{0} \text { of } x^{0} \text { and } a<0 \leqslant \leqq 1 \text { such that } \\ \text { any } \varepsilon \text {-admissible trajectory issuing from } \rho_{0} \text { whose end point belongs to } \\ V_{0} \times V_{\varepsilon} \text { is contained in } \varepsilon^{\sigma} \text {-neighborhood of } C_{\mu} \text { if } \varepsilon>0 \text { is small enough. }\end{array}\right.$

Then, there exists an initial value $G$ such that

$$
W F G=\left\{\left(y^{0}, c \eta^{0}\right) ; c>0\right\}
$$

and

$$
W F U\left(t_{0}\right) \ni \delta_{0}=\left(x^{0}, \xi^{0}\right),
$$

where $U$ is the solution of (C.P).

\section{$\S 2$. Proof of Theorem 1}

Without loss of generality we may assume that the lower order term $B$ of $L$ satisfies

$$
b_{j, j}=0, \quad b_{j, k} \neq 0 \text { if } j \neq k .
$$


Indeed, let $\alpha_{j}(t, x)$ be solutions of equations

$$
\left(D_{t}+\lambda_{j}\left(t, x, D_{x}\right)\right) \alpha_{j}+b_{j, j} \alpha_{j}=0, \quad \alpha_{j}(0, x)=1 .
$$

If we put a matrix

$$
A(t, x)=\left[\begin{array}{lll}
\alpha_{1} & & 0 \\
& \ddots & \\
0 & & \alpha_{\ell}
\end{array}\right]
$$

and put $\tilde{U}(t, x)=A^{-1}(t, x) U(t, x)$ for the solution $U$ of (C.P), we get the Cauchy problem for $\tilde{U}(t, x)$

$$
\left\{\begin{array}{l}
L_{0} \tilde{U}+A^{-1}\left(L_{0} A+B A\right) \tilde{U}=0 \\
\tilde{U}(0, x)=G, \quad G \in \mathscr{E}^{\prime},
\end{array}\right.
$$

where $L_{0}$ is the principal term of $L . \quad$ It is easy to see that $A^{-1}\left(L_{0} A+B A\right)$ satisfies the condition like $(0.4)^{\prime}$. Since the multiplier $A$ does not change the wave front set of $U$, it suffices to consider (2.1) instead of (C.P).

Let $\pi$ be the natural projection from $T^{*} R^{n}$ to $R^{n}$. If we put a transformation from $R^{n}$ to itself

$$
\pi_{j}(s, t)=\pi \chi_{j}(s, t) \pi^{-1}
$$

by means of (1.2) we get

$$
\pi_{j}(s, t) y=M_{j}(s, t) y+d_{j}(s, t)=M_{j}(t, s)^{-1}\left(y-d_{j}(t, s)\right) .
$$

We put $I_{j}(t, s) f(x)=f\left(\pi_{j}(s, t) x\right)$ for $f \in \mathscr{E}^{\prime}$ and put

$$
I(t, s)=\left[\begin{array}{ccc}
I_{1}(t, s) & 0 \\
& \ddots & \\
0 & & \dot{I}_{\ell}(t, s)
\end{array}\right] .
$$

Then, by the method of iteration we get the fundamental solution $E(t, s)$ for $L$ of the form

$$
\begin{gathered}
E(t, s)=I(t, s)+\int_{s}^{t} I(t, \theta) W(\theta, s) d \theta, \\
W(t, s)=\sum_{\nu=1}^{\infty} W_{\nu}(t, s), \quad W_{1}(t, s)=B(t) I(t, s), \\
W_{\nu+1}(t, s)=\int_{s}^{t} \int_{s}^{t_{1}} \cdots \int_{s}^{t_{\nu-1}} W_{1}\left(t, t_{1}\right) W_{1}\left(t_{1}, t_{2}\right) \cdots W_{1}\left(t_{\nu}, s\right) d t_{\nu} \cdots d t_{2} d t_{1}, \\
\nu \geqq 1 .
\end{gathered}
$$

Hence, if we denote by $\Delta_{\nu}$ and $d \tilde{t}_{\nu}$ the closure of $\Delta_{\nu}^{0}$ and $d t_{1} \cdots d t_{\nu}$, 
respectively, the solution $U\left(t_{0}, x\right)={ }^{t}\left(u_{1}\left(t_{0}, x\right), \cdots, u_{\ell}\left(t_{0}, x\right)\right)$ of (C.P) for initial value $G={ }^{t}\left(g_{1}, \cdots, g_{\ell}\right)$ is given in the form

$$
\begin{gathered}
u_{j}\left(t_{0}, x\right)=g_{j}\left(\pi_{j}\left(0, t_{0}\right) x\right)+\sum_{\nu=1}^{\infty} \sum_{\substack{j_{\nu} \in \Pi_{\nu} \\
j_{1}=j}} \int_{J_{\nu}} \beta_{J_{\nu}}^{j} g_{j_{\nu+1}}\left(\pi_{J_{\nu}}\left(\tilde{t}_{\nu}\right) x\right) d \tilde{t}_{\nu} \\
(j=1, \cdots, \ell),
\end{gathered}
$$

where

$$
\begin{aligned}
& \pi_{J_{\nu}}\left(\tilde{t}_{\nu}\right)=\pi_{j_{\nu+1}}\left(0, t_{\nu}\right) \pi_{j_{\nu}}\left(t_{\nu}, t_{\nu-1}\right) \cdots \pi_{j_{1}}\left(t_{1}, t_{0}\right), \\
& \beta_{J_{\nu}} \equiv \beta_{J_{\nu}}\left(\tilde{t}_{\nu}, x\right) \\
&=\beta_{j_{1}}\left(t_{0}, x\right) \beta_{j_{2}}\left(t_{0}, t_{1}, x\right) \cdots \beta_{j_{\nu}}\left(t_{0}, \cdots, t_{\nu-1}, x\right)
\end{aligned}
$$

and

$$
\begin{gathered}
\beta_{j_{q}}\left(t_{0}, \cdots, t_{q-1}, x\right)=-i b_{i_{q}, j_{q+1}}\left(t_{q}, \pi_{j_{q}}\left(t_{q}, t_{q-1}\right) \cdots \pi_{j_{1}}\left(t_{1}, t_{0}\right) x\right) . \\
q=1, \cdots, \nu .
\end{gathered}
$$

It follows from $(0.4)^{\prime}$ that

$$
\begin{cases}\beta_{J_{\nu}}^{j} \neq 0 & \text { if } \quad J_{\nu} \in \Pi_{\nu}^{0} \\ \beta_{J_{\nu}}^{j}=0 & \text { otherwise. }\end{cases}
$$

We take, as the initial value of (C.P),

$$
\left\{\begin{array}{l}
G={ }^{t}\left(g_{1}, \cdots, g_{\ell}\right), \quad g_{j}=g \quad(j=1, \cdots, \ell), \\
g(x)=\sum_{k=1}^{\infty} \tau_{k}^{-1} \phi\left(\tau_{k}^{1-\gamma}\left(x-y^{0}\right)\right) \exp i \tau_{k}\left(x-y^{0}\right) \cdot \eta^{0},
\end{array}\right.
$$

where $\phi \in C_{0}^{\infty}$ such that $\hat{\phi}(0)=1$ ( $\hat{\phi}$ denotes the Fourier transform of $\phi$ ). Here $r$ is a positive number such that

$$
0<\gamma<1 / 2
$$

and $\left\{\tau_{k}\right\}_{k=1}^{\infty}$ is a suitable increasing sequence of positive numbers such that $\tau_{k+1} / \tau_{k}$ is sufficiently large in order to satisfy conditions demanded later on. The function $g$ is a modification of that given by Hörmander (see Example 2.3 of [4]). Another modification was used in [10].

We have

$$
W F g=\left\{\left(y^{0}, c \eta^{0}\right) ; c>0\right\} .
$$

Indeed, since each term of $g$ is in $C^{\infty}$ and the supports shrink to $y^{0}$ it follows that 


$$
\text { sing supp } g=\left\{y^{0}\right\}
$$

Now we have

$$
\hat{g}(\xi)=\sum_{k=1}^{\infty} \tau_{k}^{(\gamma-1) n-1} \hat{\phi}\left(\left(\xi-\tau_{k} \eta^{0}\right) \tau_{k}^{\gamma-1}\right) \exp -\left(i y^{0} \cdot \xi\right) .
$$

We may assume that there exists a positive $\gamma_{0}<\gamma<1 / 2$ such that

$$
\left|\tau_{k}-\tau_{k^{\prime}}\right| \tau_{k^{\prime}}^{\gamma-1}>\tau_{k}^{\gamma_{0}} \quad \text { if } \quad k \neq k^{\prime}
$$

because we have for any integer $m>0$

$$
\begin{aligned}
\left|\tau-\tau^{\prime}\right| \tau^{\prime \gamma-1} & =\left|\tau^{1 / m}-\tau^{\prime 1 / m}\right| \sum_{r=0}^{m-1} \tau^{r / m} \tau^{\prime((m-1-r) / m)-(1-\gamma)} \\
& \geqq \tau^{[m-1-(1-\gamma) m] / m}+\sum_{r \neq[m-1-(1-\gamma) m]} \tau^{r / m} \tau^{\prime((m-1-\gamma) / m)-(1-\gamma)}
\end{aligned}
$$

if $\left|\tau-\tau^{\prime}\right|$ is large enough. Since we may assume $\sum \tau_{k}^{-1}<\infty$ and since for any $N>0$ there exists a constant $C_{N}>0$ such that

$$
\left|\hat{\phi}\left(\left(\tau_{k}-\tau_{k^{\prime}}\right) \eta^{0} \tau_{k^{\prime}}^{\gamma-1}\right)\right| \leqq C_{N} \tau_{k}^{-N} \quad \text { for } \quad k \neq k^{\prime},
$$

we have

$$
\left|\hat{g}\left(\tau_{k} \eta^{0}\right)\right| \geqq \tau_{k}^{(r-1) n-1} \hat{\phi}(0) / 2 \quad \text { if } \quad \tau_{k} \text { large enough. }
$$

Let $V$ be a conic neighborhood of $\eta^{0}$. If $\xi \notin V$, there exist two positive constants $C_{V}$ and $C_{V}^{\prime}$ such that

$$
\begin{aligned}
\left|\xi-\tau_{k} \eta^{0}\right| \tau_{k}^{\gamma-1} & \geqq C_{V}\left(|\xi|+\left|\tau_{k} \eta^{0}\right|\right) \tau_{k}^{\gamma-1} \\
& \geqq C_{V}^{\prime}|\xi|^{\gamma},
\end{aligned}
$$

where we used the Hölder inequality to obtain the second inequality. Then, for any $N>0$ there exists a constant $C_{N}^{\prime}$ such that

$$
|\hat{g}(\xi)| \leqq C_{N}^{\prime}|\xi|^{-N} \quad \text { if } \quad \xi \notin V .
$$

For the neighborhood of $V_{0}$ of $x^{0}$ given in the hypothesis (H-3) let $\psi(x)$ be $C_{0}^{\infty}\left(V_{0}\right)$ such that $\psi=1$ near $x^{0}$. For the proof of Theorem 1 it suffices to show that for some $j \in\{1, \cdots, \ell\}$

$$
\begin{aligned}
& \left|\left(\widehat{\psi} u_{j}\right)\left(t_{0}, \tau_{k} \xi^{0}\right)\right| \geqq c_{1} \tau_{k}^{(\gamma-1) n-1-\mu / 2}, \\
& c_{1}>0, \quad \text { if } \tau_{k} \text { large enough. }
\end{aligned}
$$

If we calculate the Fourier transform of $\psi u_{j}$ by (2.4) in consideration of (2.8), we get 


$$
\begin{aligned}
& \left(\widehat{\psi} u_{j}\right)\left(t_{0}, \tau_{k} \xi^{0}\right) \\
& \quad=\sum_{k^{\prime}} \tau_{k^{\prime}}^{(\gamma-1) n-1}\left(w_{(j)}\left(\tau_{k}, \tau_{k^{\prime}}\right)+\sum_{\nu=1}^{\infty} \sum_{\substack{J_{\nu} \in \Pi_{\nu}^{0} \\
j_{1}=J_{\nu}}} \int_{\Delta_{\nu}} w_{J_{\nu}}\left(\tau_{k}, \tau_{k^{\prime}} ; \tilde{t}_{\nu}\right) d \tilde{t}_{\nu}\right),
\end{aligned}
$$

where $w_{J_{q}}\left(\tau, \tau^{\prime} ; \tilde{t}_{\nu}\right)=G_{J_{\nu}}\left(\tau, \tau^{\prime} ; \tilde{t}_{\nu}\right) \exp i \tau F_{J_{\nu}}\left(\tilde{t}_{\nu}\right)\left(\nu \geqq 0, J_{0}=(j)\right)$,

$$
\begin{aligned}
& F_{J_{\nu}}\left(\tilde{t}_{\nu}\right)=-\xi^{0} \cdot\left(M_{J_{\nu}}\left(\tilde{t}_{\nu}\right) y^{0}+d_{J_{\nu}}\left(\tilde{t}_{\nu}\right)\right), \\
& G_{J_{\nu}}\left(\tau, \tau^{\prime} ; \tilde{t}_{\nu}\right)= \int \exp \left(i y \cdot\left(\tau^{\prime} \eta^{0}-\tau^{t} M_{J_{\nu}}\left(\tilde{t}_{\nu}\right) \xi^{0}\right) \tau^{\prime \gamma-1}\right) \\
& \times \psi\left(M_{J_{\nu}}\left(\tilde{t}_{\nu}\right)\left(\tau^{\prime \gamma-1} y+y^{0}\right)+d_{J_{\nu}}\left(\tilde{t}_{\nu}\right)\right) \\
& \times \phi(y) \beta_{J_{\nu}}^{\prime} d y . \quad\left(\beta_{J_{\nu}}^{\prime}=\beta_{J_{\nu}}^{j}\left|\operatorname{det} M_{J_{\nu}}\right| \neq 0\right) .
\end{aligned}
$$

Here we used the change of variables $x$ to

$$
\pi_{J_{\nu}}^{-1}\left(\tau_{k^{\prime}}^{\tau-1} y+y^{0}\right)=M_{J_{\nu}}\left(\tau_{k^{\prime}}^{\tau-1} y+y^{0}\right)+d_{J}
$$

We note that $M_{J_{\nu}}\left(\tilde{t}_{\nu}\right)$ is uniformly bounded with respect to $\nu$ and $\tilde{t}_{\nu} \in \Delta_{\nu}$ because $\pi_{J_{\nu}}\left(\tilde{t}_{\nu}\right) y$ is bounded for $|y| \leqq 1$ and so $d_{J_{\nu}}\left(\tilde{t}_{\nu}\right)$ is bounded. In the same way as in (2.14) we have for $0<\gamma_{0}<\gamma$

$$
\left|\tau^{\prime} \eta^{0}-\tau^{t} M_{J_{\nu}}\left(\tilde{t}_{\nu}\right) \xi^{0}\right| \tau^{\prime \gamma-1} \geqq \tau^{\gamma_{0}}
$$

if $\left|\tau^{\prime}-\tau\right|$ is large enough. The integration by parts with respect to $y$ shows that for any $\nu \geqq 0$ and $N>0$ there exists a constant $C_{N}$ independent of $\nu$ such that

$$
\left|G_{J_{\nu}}\left(\tau, \tau^{\prime} ; \tilde{t}_{\nu}\right)\right| \leqq C_{N}^{\nu} \tau^{-N} \quad \text { if }\left|\tau-\tau^{\prime}\right| \text { large enough. }
$$

Since the volume of $\Delta_{\nu}$ is equal to $t_{0} / \nu$ !, for the proof of (2.19) it suffices to consider only the term with respect $k^{\prime}=k$ in the sum of the right hand side of (2.20).

For $J_{\nu} \in \Pi_{\nu}^{0}(\nu \geqq 1)$ we put

$$
I_{J_{\nu}}(\tau)=\int_{\Delta_{\nu}} G_{J_{\nu}}\left(\tau ; \tilde{t}_{\nu}\right) \exp i \tau F_{J_{\nu}}(\tilde{t}) d \tilde{t}_{\nu} \quad(\nu \geqq 1),
$$

where

$$
\begin{aligned}
G_{J_{\nu}}\left(\tau ; \tilde{t}_{\nu}\right) \equiv & G_{J_{\nu}}\left(\tau, \tau ; \tilde{t}_{\nu}\right) \\
= & \int \exp \left(i \tau^{\gamma}\left(\eta^{0}-{ }^{t} M_{J_{\nu}}\left(\tilde{t}_{\nu}\right) \xi^{0}\right) \cdot y\right) \\
& \times \psi\left(M_{J_{\nu}}\left(\tilde{t}_{\nu}\right)\left(\tau^{\gamma-1} y+y^{0}\right)+d_{J_{\nu}}\left(\tilde{t}_{\nu}\right)\right) \phi(y) \beta_{J_{\nu}}^{\prime} d y .
\end{aligned}
$$


It is easy to see that for any multi-index $\alpha$ there exists a constant $C_{\alpha}$ independent of $\nu$ such that

$$
\left|\partial_{t_{\nu}}^{\alpha} G_{J_{\nu}}\left(\tau ; \tilde{t}_{\nu}\right)\right| \leqq C_{\alpha}^{\nu} \tau^{\gamma|\alpha|}, \quad 0<r<1 / 2 .
$$

When $\nu=0$ we also define $I_{J_{\nu}}(\tau)$ by the integrand of (2.26). The proof of Theorem 1 will be completed if we show the following:

Lemma 2.1. Suppose that $J_{\nu} \in \Pi_{\nu}^{0}$ and $\tau$ is large enough. If $J_{\nu} \supset J_{\mu}^{\prime}$, for any $N>0$ there exists a constant $C_{1}$ independent of $\nu$ such that

$$
\left|I_{J_{\nu}}(\tau)\right| \leqq C_{1}^{\nu} \tau^{-\mu / 2-\sigma \gamma(\nu-\mu) / 4} /(\nu-\mu) !
$$

where $\sigma$ is the positive in the hypothesis $(\mathrm{H}-3)$. If $J_{\nu} \searrow J_{\mu}^{\prime}$, for any $N>0$ there exists a constant $C_{N}$ independent of $\nu$ such that

$$
\left|I_{J_{\nu}}(\tau)\right| \leqq C_{N}^{\nu} \tau^{-N} / \nu !
$$

Furtheromore, there exists a constant $c_{0}>0$ such that

$$
\left|I_{J_{\mu}^{\prime}}(\tau)\right| \geqq c_{0} \tau^{-\mu / 2} .
$$

\section{§3. Proof of Lemma 2.1}

Suppose that $\tau$ is sufficiently large in what follows. In consideration of supports of $\phi(y)$ and $\psi\left(M_{J_{\nu}}\left(\tilde{t}_{\nu}\right)\left(\tau^{\gamma-1} y+y^{0}\right)+d_{J_{\nu}}\left(\tilde{t}_{\nu}\right)\right)$ in the right hand side of $(2.22)^{\prime}$ we see that for $\nu \geqq 1$

$$
M_{J_{\nu}}\left(\tilde{t}_{\nu}\right) y^{0}+d_{J_{\nu}}\left(\tilde{t}_{\nu}\right) \in V_{0} \quad \text { if } \quad \tilde{t}_{\nu} \in \operatorname{supp} G_{J_{\nu}} \subset \Delta_{\nu},
$$

and

$$
M_{j}\left(t_{0}, 0\right) y^{0}+d_{j}\left(t_{0}, 0\right) \in V_{0} \quad \text { if } \quad G_{(j)} \neq 0,
$$

where $V_{0}$ is the neighborhood in the hypothesis (H-3) of Theorem 1 .

First we consider the case when $\mu \geqq 1$. Because the bicharacteristic curve is admissible and because we get $(j) \neq J_{\mu}^{\prime}$, it follows from (3.1)' and the hypothesis $(\mathrm{H}-3)$ that

$$
\xi^{0} \neq{ }^{t} M_{j}\left(t_{0}, 0\right)^{-1} \eta^{0} \quad \text { if } \quad G_{(j)} \neq 0 .
$$

Hence, by means of the integration by parts with respect to $y$ we see that for any $N>0$ there exists a constant $C_{N}$ such that

$$
\left|G_{(j)}\right| \leqq C_{N} \tau^{-N}
$$

which gives the inequality (2.29) with $\nu=0$. 
Let $\kappa>0$ be a small parameter chosen later on. Let $h(s)$ be a $C^{\infty}$ function such that $0 \leqq h \leqq 1, h=1$ for $s \leqq 1$ and $h=0$ for $s \geqq 2$. If we put

$$
\psi_{J_{\nu}}\left(\tilde{t}_{\nu}\right)=h\left(\kappa^{-3_{\nu}} \tau^{\gamma / 2}\left|\eta^{0}-{ }^{t} M_{J_{\nu}}\left(\tilde{t}_{\nu}\right) \xi^{0}\right|\right), \quad \nu \geqq 1,
$$

the integration by parts with respect to $y$ shows that

$$
\left|G_{J_{\nu}}\left(\tau ; \tilde{t}_{\nu}\right)\right| \leqq C_{N}^{\nu} \tau^{-N} \quad \text { if } \quad \tilde{t}_{\nu} \in \operatorname{supp}\left(1-\psi_{J_{\nu}}\right)
$$

because we have

$$
\left|\eta^{0}-{ }^{t} M_{J_{\nu}}\left(\tilde{t}_{\nu}\right) \xi^{0}\right| \geqq \kappa^{3 \nu} \tau^{-r / 2} \quad \text { on } \operatorname{supp}\left(1-\psi_{J_{\nu}}\right) \text {. }
$$

Since the volume of $\Delta_{\nu}$ is $t_{0} / \nu$ !, in view of (3.5) it suffices to consider $G_{J_{\nu}}^{\prime}$ $=\psi_{J_{\nu}} G_{J_{\nu}}$ in place of $G_{J_{\nu}}$ in (2.26). For $G_{J_{\nu}}^{\prime}$ we have the inequality of the same type as (2.27).

If $\tilde{t}_{\nu} \in \operatorname{supp} G_{J_{\nu}}^{\prime} \cap \Delta_{\nu}$ then the end point of $C\left(J_{\nu}, \tilde{t}_{\nu}, \rho_{0}\right)$ belongs to $V_{0}$ $\times V_{\varepsilon}$ with $\varepsilon=\kappa^{\nu} \tau^{-\gamma / 2}$. Indeed, it is the consequence of (3.1) and

$$
\left|{ }^{t} M_{J_{\nu}}^{-1} \eta^{0}-\xi^{0}\right| \leqq \kappa^{2 \nu} \tau^{-\gamma / 2} \quad \text { on } \operatorname{supp} \psi_{J_{\nu}},
$$

where we used ${ }^{t} M_{J_{\nu}}^{-1} \kappa^{\nu} \ll 1$ for $\kappa$ small enough.

Let $E\left(J_{\nu}\right)$ be a subset of $\Delta_{\nu}$ defined by

$$
E\left(J_{\nu}\right)=\left\{\tilde{t}_{\nu} \in \operatorname{supp} G_{J_{\nu}}^{\prime} \cap A_{\nu} ; C\left(J_{\nu}, \tilde{t}_{\nu}, \rho_{0}\right) \text { is } \kappa^{\nu} \tau^{-\gamma / 2} \text {-admissible }\right\} .
$$

It follows from $(\mathrm{H}-3)$ that if $J_{\nu} \triangleright J_{\mu}^{\prime}$ then $E\left(J_{\nu}\right)=\emptyset$. In the case $J_{\nu} \supset J_{\mu}^{\prime}$, if $\tilde{t}_{\nu} \in E\left(J_{\nu}\right)$ we have (1.6) and (1.7) with $\varepsilon_{0}=\left(\kappa^{\nu} \tau^{-r / 2}\right)^{\sigma}$. We note that the number of possibilities of subset $\left\{q_{1}, \cdots, q_{\mu}\right\}\left(\equiv Q_{\mu}\right)$ satisfying (1.6) is inferior to $2^{\nu}$. We denote by $Q_{\mu}^{j}\left(j=1, \cdots, n_{\nu}, n_{\nu} \leqq 2^{\nu}\right)$ all such subsets. For a fixed subset $Q_{\mu}=\left\{q_{1}, \cdots, q_{\mu}\right\}$ let $q_{j}^{\prime}\left(j=1, \cdots, n_{\nu}^{\prime}\right)$ be all elements of $\{1, \cdots, \nu+1\}$ satisfying (1.8). Clearly, the number $n_{\nu}^{\prime}$ is superior to $[(\nu-\mu+1) / 2]$. We put

$$
\phi_{J_{\nu}}\left(t_{\nu}\right)=\sum_{r=1}^{\mu} h\left(\left|t_{q_{r}}-t_{r}^{\prime}\right| / \varepsilon_{0}\right) \sum_{j=1}^{n_{\nu}^{\prime}} h\left(\left|t_{q_{j}^{\prime}}-t_{q_{j}^{\prime}-1}\right| / \varepsilon_{0}\right),
$$

where $\varepsilon_{0}=\left(\kappa^{\nu} \tau^{-r / 2}\right)^{\sigma}$. For each $Q_{\mu}^{j}$ if we define $\phi_{J_{\nu}}^{j}$ similarly, it follows from (H-3) that

$$
E\left(J_{\nu}\right) \cap\left(\bigcap_{j=1}^{n_{\nu}} \operatorname{supp}\left(1-\phi_{J_{\nu}}^{j}\right)\right)=\emptyset .
$$

For a moment we suppose $J_{\nu} \supset J_{\mu}^{\prime}$. Put 


$$
\left\{\begin{array}{l}
G_{J_{\nu}}^{1}=G_{J_{\nu}}^{\prime} \phi_{J_{\nu}}^{1}, \quad G_{J_{\nu}}^{j}=G_{J_{\nu}}^{\prime} \phi_{J_{\nu}}^{j} \prod_{m=1}^{j-1}\left(1-\phi_{J_{\nu}}^{m}\right), \quad 2 \leqq j \leqq n_{\nu}, \\
G_{J_{\nu}}^{\prime \prime}=G_{J_{\nu}}^{\prime} \prod_{m=1}^{n_{\nu}}\left(1-\phi_{J_{\nu}}^{m}\right) .
\end{array}\right.
$$

For $G_{J}^{j}$ and $G_{J}^{\prime \prime}$ we have the inequalities of the same type as (2.27). We have a decomposition

$$
I_{J_{\nu}}^{\prime}(\tau)=\sum_{j=1}^{n_{\nu}} I_{J_{\nu}}^{j}(\tau)+I_{J_{\nu}}^{\prime \prime}(\tau)
$$

where $I_{J_{\nu}}^{\prime}, I_{J_{\nu}}^{j}, I_{J_{\nu}}^{\prime \prime}$ are defined by (2.26) with $G_{J_{\nu}}$ replaced by $G_{J_{\nu}}^{\prime}, G_{J_{\nu}}^{j}, G_{J_{\nu}}^{\prime \prime}$, respectively. It follows from (3.8) that if $\tilde{t}_{\nu} \in \operatorname{supp} G_{J_{\nu}}^{\prime \prime}$ then there exists a $q \in\{1, \cdots, \nu\}$ such that

$$
\left|\partial_{t_{q}} F_{J}\left(\tilde{t}_{\nu}\right)\right| \geqq \kappa^{\nu} \tau^{-\gamma / 2} / 2 .
$$

In order to show this inequality we prepare a proposition which is the immediate consequence of Theorem 2.3 of Kumano-go-Taniguchi-Tozaki [8].

Proposition 3.1. Let $\phi_{j}(t, s)=\phi_{j}(t, s ; x, \xi)$ be solutions of eiconal equations

$$
\partial_{t} \phi_{j}+\lambda_{j}\left(t, x, d_{x} \phi_{j}\right)=0,\left.\quad \phi_{j}(t, s)\right|_{t=s}=x \cdot \xi \quad j=1, \cdots, \ell .
$$

For a sequence of phase-functions

$$
\phi_{j_{1}}\left(t_{0}, t_{1}\right), \quad \phi_{j_{2}}\left(t_{1}, t_{2}\right), \cdots, \phi_{J_{\nu+1}}\left(t_{\nu}, 0\right)
$$

$\left(J_{\nu}=\left(j_{1}, \cdots, j_{\nu+1}\right), \tilde{t}_{\nu} \in \Delta_{\nu}\right)$ if we denote by $\Phi_{J_{\nu}}\left(t_{0}, \tilde{t}_{\nu}, 0 ; x, \xi\right)$ the multi-product

$$
\left(\phi_{j_{1}}\left(t_{0}, t_{1}\right) \# \phi_{j_{2}}\left(t_{1}, t_{2}\right) \# \cdots \# \phi_{j_{\nu+1}}\left(t_{\nu}, 0\right)\right)(x, \xi),
$$

then we get

$$
\left.\partial_{t_{q}} \Phi_{J_{\nu}}\left(t_{0}, \tilde{t}_{\nu}, 0 ; x, \xi\right)\right|_{\substack{x=z^{\nu} \\ \xi=\eta}}=\left(\lambda_{j_{q}}-\lambda_{j_{q+1}}\right)\left(t_{q}, x^{q}, \xi^{q}\right), \quad q=1, \cdots, \nu,
$$

where

and

$$
\left(x^{q}, \xi^{q}\right)=\chi_{j_{q+1}}\left(t_{q}, t_{q+1}\right) \cdots \chi_{j_{\nu+1}}\left(t_{\nu}, 0\right)(y, \eta)
$$

$$
z^{\nu}=\pi \chi_{j_{1}}\left(t_{0}, t_{1}\right) \ldots \chi_{j_{\nu+1}}\left(t_{\nu}, 0\right)(y, \eta) .
$$

Because $\phi_{j}(t, s ; x, \xi)=\left(\pi_{j}(s, t) x\right) \cdot \xi=M_{j}^{-1}(t, s)\left(x-d_{j}(t, s)\right) \cdot \xi$ we have

$$
\begin{aligned}
\Phi_{J_{\nu}}\left(t_{0}, \tilde{t}_{\nu}, 0 ; x, \xi\right) & =\left(\pi_{J_{\nu}}\left(\tilde{t}_{\nu}\right) x\right) \cdot \xi \\
& =M_{J_{\nu}}\left(t_{\nu}\right)^{-1}\left(x-d_{J_{\nu}}\left(\tilde{t}_{\nu}\right)\right) \cdot \xi .
\end{aligned}
$$


Then we get

$$
\left.\partial_{t_{q}} \Phi_{J_{\nu}}\right|_{\substack{x=M_{j} J_{\nu} y^{0}+d_{J_{\nu}} \\ \xi=\eta^{0}}}=\left(\xi^{0}-{ }^{t} M_{J_{\nu}}^{-1} \eta^{0}\right) \cdot \partial_{t_{q}}\left(d_{J_{\nu}}+M_{J_{\nu}} y^{0}\right)+\partial_{t_{q}} F_{J_{\nu}} .
$$

In view of (3.13) and (3.6) we get (3.11) because the first term of the right hand side of (3.14) is smaller than $\kappa^{\nu} \tau^{-\gamma / 2} / 2$ if we choose $\kappa$ sufficiently small.

We shall show that for any $N>0$ there exists a constant $C_{N}$ such that

$$
\left|I_{J_{\nu}}^{\prime \prime}(\tau)\right| \leqq C_{N}^{\nu} \tau^{-N} / \nu !
$$

For a fixed $\tau$, let $\sum_{j} \psi_{J_{\nu}}^{j}\left(\tilde{t}_{\nu}\right) \equiv 1$ be a partition of unity on $\operatorname{supp} G_{J_{\nu}}^{\prime \prime} \cap \Delta_{\nu}$ such that the number of $\psi_{J_{\nu}}^{j}$ whose supports superpose each other is inferior to $2^{\nu}$ and we have

$$
\begin{gathered}
\left|\tilde{t}_{\nu}-\tilde{t}_{\nu}^{\prime}\right| \leqq \kappa^{2 \nu} \tau^{-\gamma / 2} \quad \text { if } \tilde{t}_{\nu}, \tilde{t}_{\nu}^{\prime} \in \operatorname{supp} \psi_{J_{\nu}}^{j}, \\
\left|\partial_{t_{\nu}}^{\alpha} \psi_{J_{\nu}}^{j}\right| \leqq C_{\alpha}^{\nu} \tau^{\gamma|\alpha| / 2}
\end{gathered}
$$

for a constant $C_{\alpha}$ independent of $\tau$ and $\nu$. In fact, we can make such a partition of unity by putting

$$
\psi_{J}^{j}=h_{j} / \sum_{j} h_{j}, \quad h_{j}\left(\tilde{t}_{\nu}\right)=h\left(\left|\tilde{t}_{\nu}-\tilde{t}_{\nu}^{j}\right| \kappa^{-2 \nu} \tau^{\gamma / 2}\right)
$$

for suitable points $\tilde{t}_{\nu}^{j} \in \operatorname{supp} G_{J}^{\prime \prime} \cap \Delta_{\nu}$. By (3.11) and (3.16) we see that

$$
\left|\partial_{t_{q}} F_{J_{\nu}}\right| \geqq \kappa^{\nu} \tau^{-\gamma / 2} / 3 \quad \text { on } \quad \operatorname{supp} \psi_{J_{\nu}}^{j}
$$

because $\kappa^{2 \nu} \ll \kappa^{\nu}$. Note that

$$
\int_{\Delta_{\nu}} d t_{\nu}=\int_{\Delta_{\nu-1}} d t_{1} \cdots d t_{q-1} d t_{q+1} \cdots d t_{\nu} \int_{t_{q+1}}^{t_{q-1}} d t_{q} .
$$

By means of (3.18) it follows from the integration by parts that

$$
\begin{gathered}
\int_{t_{q+1}}^{t_{q-1}} e^{i \tau F_{\nu}} \psi_{\nu}^{j} G_{\nu}^{\prime \prime} d t_{q}=\left[-i \tau^{-1} e^{i \tau F_{\nu}} \psi_{\nu}^{j} G_{\nu}^{\prime \prime}\left(\partial_{t_{q}} F_{\nu}\right)^{-1}\right]_{t_{q}=t_{q+1}}^{t_{q}=t_{q-1}} \\
\quad+i \tau^{-1} \int_{t_{q+1}}^{t_{q-1}} e^{i \tau F_{\nu}} \partial_{t_{q}}\left(\psi_{\nu}^{j} G_{\nu}^{\prime \prime}\left(\partial_{t_{q}} F_{\nu}\right)^{-1}\right) d t_{q}
\end{gathered}
$$

where for the brevity we wrote $\nu$ instead of $J_{\nu}$. Noting that

$$
\left.F_{J_{\nu}}\left(\tilde{t}_{\nu}\right)\right|_{t_{q}=t_{q+1}}=F_{J_{\nu-1}^{q}}\left(t_{1}, \cdots, t_{q-1}, t_{q+1}, \cdots, t_{\nu}\right)
$$

for $J_{\nu-1}^{q}=\left(j_{1}, \cdots, j_{q-1}, j_{q+1}, \cdots, j_{\nu+1}\right)$, in view of (3.17)-(3.20) we have 


$$
I_{J_{\nu}}^{\prime \prime}(\tau)=\tau^{\gamma / 2-1}\left(\sum_{q} \tilde{I}_{J_{\nu-1}^{q}}(\tau)+\tau^{\tau} \tilde{I}_{J_{\nu}}(\tau)\right),
$$

where the first term of the right hand side is the sum with respect to all $q$ satisfying (3.18) for each $\psi_{J_{\nu}}^{j}$. Here $\tilde{I}_{J_{\nu-1}^{q}}(\tau), I_{J_{\nu}}(\tau)$ are defined, as in (2.26), with phases $F_{J_{\nu}^{q}-1}, F_{J_{\nu}}$ and amplitudes $G_{J_{\nu}^{q}-1}, \tilde{G}_{J_{\nu}}$ satisfying inequalities of the same type as (2.27), respectively, because the number of the superposition of $\psi_{J_{\nu}}^{j}$ is inferior to $2^{\nu}$ and because we have (3.17) and (3.18). If $j_{q-1} \neq j_{q+1}$ in $J_{\nu-1}^{q}$, we can use the above procedure for $\tilde{I}_{J_{\nu-1}^{q}}(\tau)$ instead of $I_{J_{\nu}}^{\prime \prime}(\tau)$ because we have the inequality similar to (3.11) if $\tilde{t}_{\nu-1} \in \operatorname{supp} \tilde{G}_{J_{\nu-1}^{g}}$ $\cap \Delta_{\nu-1}$. Note that for $J_{\nu} \in \Pi_{\nu}$ we have

$$
F_{J_{\nu}}\left(\tilde{t}_{\nu}\right)=F_{J_{\nu-1}^{\prime}}\left(\tilde{t}_{\nu-1}\right) \quad \text { if } j_{q}=j_{q+1},
$$

where $J_{\nu-1}^{\prime}$ and $\tilde{t}_{\nu-1}$ are defined by removing $j_{q}$ and $t_{q}$ in $J_{\nu}$ and $\tilde{t}_{\nu}$, respectively. If we define (2.26) also for $\left.J_{\nu} \in \Pi_{\nu}\right\rangle \Pi_{\nu}^{0}$ then we get

$$
I_{J_{\nu}}(\tau)=I_{J_{\nu-1}^{\prime}}(\tau) \quad \text { if } j_{q}=j_{q+1},
$$

where the amplitude of $I_{J_{\nu-1}^{\prime}}(\tau)$ is given in the form

$$
\int_{t_{q+1}}^{t_{q-1}} G_{J_{\nu}}\left(\tau, \tilde{t}_{\nu}\right) d t_{q}
$$

It follows from the formula (3.23) with $J_{\nu}=J_{\nu-1}^{q}$ that we can use the above procedure also for $I_{J_{\nu-1}^{q}}(\tau)$ when $j_{q-1}=j_{q+1}$ in $J_{\nu-1}^{q}$. Repeating the integration by parts with respect to $t_{q}$, in view of $3 r / 2-1<0$ we get (3.15) because (2.29) with $\nu=0$ is verified.

When $J_{\nu} \ngtr J_{\mu}^{\prime}$, the method which gives (3.15) is applicable to show (2.29) for $I_{J_{\nu}}(\tau)$ because $E\left(J_{\nu}\right)=\emptyset$ and so we have (3.11) if $\tilde{t}_{\nu} \in \operatorname{supp} G_{J_{\nu}}^{\prime}$.

To get (2.28) it suffices to show

$$
\left|I_{J}^{j}(\tau)\right| \leqq C^{\nu} \tau^{-\mu / 2-\sigma_{\gamma}(\nu-\mu) / 4} / \nu !
$$

for a constant $C$ independent of $\tau$ and $\nu$, because $n_{\nu} \leqq 2^{\nu}$. We consider a $I_{j}^{j}(\tau)$. For the simplicity we write $j=0$. Noting the hypothesis (H-3) and support of $\phi_{J_{\nu}}$ defined by (3.7), we can see that there exist two subsets $\left\{p_{1}, \cdots, p_{\mu}\right\}$ and $\left\{p_{1}^{\prime}, \cdots, p_{\mu}^{\prime}\right\}$ of $\{1, \cdots, \nu\}$ such that

$$
\left\{\begin{array}{l}
p_{j} \leqq q_{j} \leqq p_{j}^{\prime} \quad(j=1, \cdots, \mu) \\
p_{j}^{\prime}<p_{j+1} \quad(j=1, \cdots, \mu-1)
\end{array}\right.
$$

and with $\varepsilon_{0}=\left(\kappa^{\nu} \tau^{-r / 2}\right)^{\sigma}$ we have 


$$
\begin{gathered}
\left|t_{p_{r}}-t_{p_{r}^{\prime}}\right| \leqq \nu \varepsilon_{0}, \quad r=1, \cdots, \mu \\
\left\{\begin{array}{l}
\left|t_{p_{r}}-t_{p_{r}-1}\right| \geqq \varepsilon_{0} \\
\left|t_{p_{r}^{\prime}}-t_{p_{r+1}^{\prime}}\right| \geqq \varepsilon_{0} \quad r=1, \cdots, \mu, \quad t_{\nu+1}=0 \\
\text { if } \tilde{t}_{\nu} \in \operatorname{supp} G_{J_{\nu}}^{0} \cap \Delta_{\nu} .
\end{array}\right.
\end{gathered}
$$

Note that the number of possibilities of subsets $\left\{p_{1}, \cdots, p_{\mu}\right\}\left(\equiv P_{\mu}\right)$ and $\left\{p_{1}^{\prime}, \cdots, p_{\mu}^{\prime}\right\}\left(\equiv P_{\mu}^{\prime}\right)$ is inferior to $4^{\nu}$. We denote by $\left(P_{\mu}^{j}, P_{\mu}^{\prime j}\right)\left(j=1, \cdots, n_{\nu}^{\prime \prime}\right.$, $\left.n_{\nu}^{\prime \prime} \leqq 4^{\nu}\right)$ all couples of two subsets.

For a $\left(P_{\mu}, P_{\mu}^{\prime}\right)$ we put

$$
f_{J_{\nu}}\left(\tilde{t}_{\nu}\right)=\prod_{r=1}^{\mu}(1-h)\left(\left|t_{p_{r}}-t_{p_{r}-1}\right| / \varepsilon_{0}\right)(1-h)\left(\left|t_{p_{r}^{\prime}}-t_{p_{r}^{\prime}+1}\right| / \varepsilon_{0}\right) .
$$

If we define $f_{J_{\nu}}^{j}$ for each $\left(P_{\mu}^{j}, P_{\mu}^{\prime j}\right)$ similarly as $f_{J_{\nu}}$, then we get by means of (H-3)

$$
\operatorname{supp} G_{J_{\nu}}^{0} \cap \Delta_{\nu} \cap\left(\bigcap_{j=1}^{n_{\nu}^{\prime \prime}} \operatorname{supp}\left(1-f_{J_{\nu}}^{j}\right)\right)=\emptyset .
$$

Then we have a decomposition

$$
I_{J_{\nu}}^{0}(\tau)=\sum_{j=1}^{n_{\nu}^{\prime \prime}} I_{J_{\nu}}^{0, j}(\tau)
$$

where $I_{J_{\nu}}^{0, j}$ are defined by (2.26) with $G_{J_{\nu}}$ replaced by

$$
G_{J_{\nu}}^{0,1}=G_{J_{\nu}}^{0} f_{J_{\nu}}^{1}, \quad G_{J_{\nu}}^{0, j}=G_{J_{\nu}}^{0} f_{J_{\nu}}^{j} \sum_{m=1}^{j-1}\left(1-f_{J_{\nu}}^{m}\right), \quad j \geqq 2,
$$

for which we have inequalities of the same type as (2.26).

We consider a $I_{J_{\nu}}^{0, j}(\tau)$. For the brevity we write $j=0$. We take the change of variables $\tilde{t}_{\nu}=\left(t_{1}, \cdots, t_{\nu}\right)$ to $\left(\tilde{s}_{\mu}, \tilde{w}_{\nu_{1}}, \tilde{v}_{\nu_{2}}\right)$ as follows: We put

$$
\begin{aligned}
& \tilde{s}_{\mu}=\left(s_{1}, \cdots, s_{\mu}\right) \\
& s_{r}=\left(t_{p_{r}}+t_{p_{r+1}}+\cdots+t_{r_{r}^{\prime}}\right) /\left(p_{r}^{\prime}-p_{r}+1\right) .
\end{aligned}
$$

For any pair $(q, q-1)(q=1, \cdots, \nu)$ except $\left(p_{r}, p_{r}-1\right)(r=1, \cdots, \mu)$ we put

$$
\left\{\begin{array}{l}
w_{q}=t_{q-1}-t_{q} \quad \text { if } \quad\left|t_{q-1}-t_{q}\right| \leqq \nu \varepsilon_{0} \quad \text { on } \quad \operatorname{supp} G_{J_{\nu}}^{0,0} \\
v_{q}=t_{q-1}-t_{q} \quad \text { otherwise }
\end{array}\right.
$$

and put

$$
\tilde{w}_{\nu_{1}}=\left(w_{1}, \cdots, w_{\nu_{1}}\right), \quad \tilde{v}_{\nu_{2}}=\left(v_{1}, \cdots, v_{\nu_{2}}\right)
$$


It follows from (3.7) that

$$
\nu_{1} \geqq n_{\nu}^{\prime} \geqq[(\nu-\mu+1) / 2] .
$$

By the above change of variables we get

$$
I_{J_{\nu}}^{0,0}(\tau)=\int_{D_{1}} d \tilde{w}_{\nu_{1}} \int_{D_{2}} d \tilde{v}_{\nu_{2}} \int \tilde{G}_{J_{\nu}}^{0,0} \exp i \tau \tilde{F}_{J_{\nu}} d \tilde{s}_{\mu}
$$

where $\tilde{G}_{J_{\nu}}^{0,0}\left(\tau ; \tilde{s}_{\mu}, \tilde{w}_{\nu_{1}}, \tilde{v}_{\nu_{2}}\right)=G_{J_{\nu}}^{0,0}\left(\tau ; \tilde{t}_{\nu}\right)$ and $\tilde{F}_{J_{\nu}}\left(\tilde{s}_{\mu}, \tilde{w}_{\nu_{1}}, \tilde{v}_{\nu_{2}}\right)=F_{J_{\nu}}\left(\tilde{t}_{\nu}\right)$. Here

$$
\begin{aligned}
& D_{1}=\left\{w_{j} ; 0 \leqq w_{j} \leqq \nu\left(\kappa^{\nu} \tau^{-r / 2}\right)^{\sigma}\right\}, \\
& D_{2}=\left\{v_{j} \geqq 0 ; \sum_{j=1}^{\nu_{2}} v_{j} \leqq t_{0}\right\} .
\end{aligned}
$$

In view of (3.21) and (3.22) it follows from (H-3) that

$$
\tilde{F}_{J_{\nu}}\left(\tilde{s}_{\mu}, 0, \tilde{v}_{\nu_{2}}\right)=F_{J_{\mu}^{\prime}}\left(\tilde{s}_{\mu}\right) \text {. }
$$

Noting (3.13) and (3.14), we can see by (H-2) and (H-3) that

$$
\tilde{s}_{\mu}=\tilde{t}_{\mu}^{\prime} \Longleftrightarrow \partial_{s_{q}} F_{J_{\mu}^{\prime}}\left(\tilde{s}_{\mu}\right)=0 \quad \text { for } \quad q=1, \cdots, \mu,
$$

if $\tilde{s}_{\mu} \in \pi^{*} \operatorname{supp} \tilde{G}_{J_{\nu}}^{0,0}$, where $\pi^{*}$ is the natural projection from $R_{\tilde{s}} \times R_{\widetilde{w}} \times R_{\tilde{v}}$ to $R_{\tilde{s}}$. By $(\mathrm{H}-2)$ we get

$$
\operatorname{det}\left(\partial_{s_{p}} \partial_{s_{q}} \tilde{F}_{J_{\nu}}\left(\tilde{s}_{\mu}, \tilde{w}_{\nu_{1}}, \tilde{v}_{\nu_{2}}\right)\right) \neq 0 \quad \text { on } \quad \operatorname{supp} \tilde{G}_{J_{\nu}}^{0,0} \cap R_{\tilde{s}} \times D_{1} \times D_{2}
$$

if we choose $\kappa$ small enough. On account of (3.33) and (3.34), the implicit function theorem shows the existence of the unique solution $\tilde{s}_{\mu}\left(\tilde{w}_{\nu_{1}}, \tilde{v}_{\nu_{2}}\right)$ of the equation

$$
\partial_{s_{q}} \tilde{F}_{J_{\nu}}\left(\cdot, \tilde{w}_{\nu_{1}}, \tilde{v}_{\nu_{2}}\right)=0, \quad q=1, \cdots, \mu
$$

if $\left(\tilde{w}_{\nu_{1}}, \tilde{v}_{\nu_{2}}\right)$ varies on $D_{1} \times D_{2}$. Regarding $\left(\tilde{w}_{\nu_{1}}, \tilde{v}_{\nu_{2}}\right)$ as parameters we see by means of the stationary phase method (see $\S 1-2$ of [2]) that

$$
\begin{aligned}
I_{J_{\nu}}(\tau ; \tilde{w}, \tilde{v})= & \int \tilde{G}_{J_{\nu}}^{0,0} \exp i \tau \tilde{F}_{J_{\nu}} d \tilde{s}_{\mu} \sim(2 \pi / \tau)^{\mu / 2}|\operatorname{det} Q|^{-1 / 2} e^{(\pi i / 4) \operatorname{sgn} Q} \\
& \times \sum_{j=0}^{\infty}\left(R^{j} \tilde{G}_{J_{\nu}}^{0,0}\right) \tau^{-j} / j !, \quad \tau \rightarrow \infty
\end{aligned}
$$

where

$$
\begin{aligned}
Q & \equiv Q(\tilde{w}, \tilde{v}) \\
& =\left(\partial_{s_{p}} \partial_{s_{q}} \tilde{F}_{J_{\nu}}\left(\tilde{s}_{\mu}(\tilde{w}, \tilde{v}), \tilde{w}, \tilde{v}\right) ; \begin{array}{l}
p=1, \cdots, \mu \rightarrow \\
q=1, \cdots, \mu \downarrow
\end{array}\right),
\end{aligned}
$$


$R=i<Q^{-1} \partial_{\tilde{s}_{\mu}^{\prime}}, \partial_{\tilde{s}_{\mu}^{\prime}}>/ 2$ and $\widetilde{\widetilde{G}}_{J_{\nu}}^{0,0}\left(\tilde{s}_{\mu}^{\prime}, \tilde{w}, \tilde{v}\right)=\tilde{G}_{J_{\nu}}^{0,0}\left(\tilde{s}_{\mu}, \tilde{w}, \tilde{v}\right) . \quad \operatorname{Here}\left(\tilde{s}_{\mu}, \tilde{w}, \tilde{v}\right) \rightarrow$ $\tilde{s}_{\mu}^{\prime}$ is a $C^{\infty}$ mapping such that

$$
\begin{aligned}
& \tilde{s}_{\mu}^{\prime}\left(\tilde{s}_{\mu}, \tilde{w}, \tilde{v}\right)=\tilde{s}_{\mu}-\tilde{s}_{\mu}(\tilde{w}, \tilde{v})+0\left(\left|\tilde{s}_{\mu}-\tilde{s}_{\mu}(\tilde{w}, \tilde{v})\right|^{2}\right), \\
& \tilde{F}_{J_{\nu}}\left(\tilde{s}_{\mu}, \tilde{w}, \tilde{v}\right)=\tilde{F}_{J_{\nu}}\left(\tilde{s}_{\mu}(\tilde{w}, \tilde{v}), \tilde{w}, \tilde{v}\right)+<Q \tilde{s}_{\mu}^{\prime}, \tilde{s}_{\mu}^{\prime}>/ 2 .
\end{aligned}
$$

Since volumes of $D_{1}$ and $D_{2}$ are estimated by $\tau^{-\sigma \gamma_{1} / 2} / \nu_{1} !$ and $t_{0}^{\nu_{2}} / \nu_{2}$ ! respectively, in view of (3.35) and (3.30) it is easy to see that $I_{J \nu}^{0,0}(\tau)$ is inferior to the right hand side of (3.24). It follows from (3.29) that we get (3.24), which shows (2.28).

When $J_{\nu}=J_{\mu}^{\prime}$ we note that $n_{\nu}$ of (3.10) and $n_{\nu}^{\prime \prime}$ of (3.29) are 1 and that the change of variables $\tilde{t}_{\nu} \rightarrow\left(\tilde{s}_{\mu}, \tilde{w}_{\nu_{1}}, \tilde{v}_{\nu_{2}}\right)$ is not necessary, that is, $\tilde{s}_{\mu}=\tilde{t}_{\mu}$. Hence the formula (3.35) gives (2.30) if we show that there exists a constant $c_{1}>0$ such that

$$
\left|G_{J_{\mu}^{\prime}}\left(\tau ; \tilde{t}_{\mu}^{\prime}\right)\right| \geqq c_{1}>0 \text {. }
$$

Since $\xi^{0}={ }^{t} M_{J_{\mu}^{\prime}}\left(\tilde{t}_{\mu}^{\prime}\right) \eta^{0}$ it follows from $(2.22)^{\prime}$ that

$$
G_{J_{\mu}^{\prime}}\left(\tau ; \tilde{t}_{\mu}^{\prime}\right)=\int \psi\left(M_{J_{\mu}^{\prime}}\left(\tilde{t}_{\mu}^{\prime}\right)\left(\tau^{\gamma-1} y+y^{0}\right)+d_{J_{\mu}^{\prime}}\left(\tilde{t}_{\mu}^{\prime}\right)\right) \phi(y) \beta_{J_{\nu}}^{\prime} d y .
$$

Noting (2.8) and the change of variables (2.23), we see that $\beta_{J_{\nu}}^{j}$ is almost equal to a non-zero constant on $\operatorname{supp} \phi$ when $\tau$ is sufficiently large (note the argument of $\psi$ in (3.37)). Then we get (3.36) if $\tau$ is large enough.

We consider the case when $\mu=0$. If $J_{\mu}^{\prime}=(j)$, that is, the admissible trajectory $C_{\mu}$ is bic. curve w.r.t., $\lambda_{j}$ then there exists a constant $c_{2}>0$ such that

$$
\left|G_{(j)}(\tau)\right| \geqq c_{2}>0 \quad \text { if } \tau \text { large enough, }
$$

which shows (2.30). Inequality (2.29) is obtained in the same manner as in the case $\mu \geqq 1$. If we replace the definition of $\phi_{J_{\nu}}\left(\tilde{t}_{\nu}\right)$ by

$$
\phi_{J \nu}\left(\tilde{t}_{\nu}\right)=\prod_{j=1}^{n_{\nu}^{\prime}} h\left(\left|t_{q_{j}^{\prime}}-t_{q_{j-1}^{\prime}}\right| / \varepsilon_{0}\right) \quad \text { with } \quad \varepsilon_{0}=\left(\kappa^{\nu} \tau^{-r / 2}\right)^{\sigma},
$$

the second term of the right hand side of (3.10) is estimated by the right hands side of (2.29), and the first term of the right hand side of (3.10) estimated by the right hand side of (2.28) because the volume of $\Delta_{\nu} \cap$ $\operatorname{supp} \phi_{J_{\nu}}$ is inferior to $t_{0}^{\nu} \tau^{-\sigma \gamma \nu} / \nu$ ! Then we have (2.28) when $\mu=0$.

Remark 1. It follows from Lemma 2.1 that if $U$ is the solution of 
(C.P) for the initial value $G$ defined by (2.9) we have

$$
\begin{gathered}
c_{3} \leqq\left|(\widehat{\psi} U)\left(t_{0} ; \tau_{k} \xi^{0}\right)\right| \tau^{(1-\gamma) n+1+\mu / 2} \leqq c_{3}^{-1} \\
\text { if } \quad k \geqq k_{0}, \quad k_{0} \text { large enough, }
\end{gathered}
$$

where the function $\psi \in C_{0}^{\infty}\left(V_{0}\right)$ was taken before (2.19) and $c_{3}$ is a positive constant independent of $\tau_{k}$. By means of the formula (3.35) and the argument in getting (3.36) we see that

$$
c_{3}=c_{4}^{\mu}\left|\operatorname{det}\left(\partial_{t_{p}} \partial_{t_{q}} \xi^{0} \cdot\left(M_{J_{\mu}^{\prime}}\left(\tilde{t}_{\mu}^{\prime}\right) y^{0}+d_{J_{\mu}^{\prime}}(\tilde{t})\right)\right)\right|^{-1 / 2},
$$

where $c_{4}$ is a positive constant independent of $\mu$ and the choice of $\psi \in$ $C_{0}^{\infty}\left(V_{0}\right)$ such that $\psi=1$ near $x^{0}$. We note that the number $k_{0}$ of (3.38) depends on the choice of $\psi$.

Remark 2. The proof of Theorem 1 is also valid when there exist plural admissible trajectories which link $\rho_{0}$ with $\delta_{0}=\left(x^{0}, \xi^{0}\right)$ if numbers of step are different each other, the condition (H-2) is satisfied for each adm. traj. and the following condition is verified:

$$
\left\{\begin{array}{l}
\text { Let } V_{\varepsilon} \text { be a } \varepsilon \text {-conic neighborhood of } \xi^{0} \text {. There exist an open } \\
\text { neighborhood } V_{0} \text { of } x^{0} \text { and } 0<\sigma \leqq 1 \text { such that any } \varepsilon \text {-admissible } \\
\text { trajectory issuing from } \rho_{0} \text { whose end point belongs to } V_{0} \times V_{\varepsilon} \text { is } \\
\text { contained in } \varepsilon^{\sigma} \text {-neighborhood of some admissible trajectory given } \\
\text { above if } \varepsilon>0 \text { small enough. }
\end{array}\right.
$$

Indeed, it is clear if we note that the contribution with respect to the admissible trajectory of the smallest step is dominant.

\section{§4. Application of Theorem 1}

We consider an example of $L$ with $l=2$ on $R_{t}^{1} \times R_{x}^{1}$ whose $\lambda_{1}$ and $\lambda_{2}$ are defined

$$
\lambda_{1}=\alpha(t) D_{x}, \quad \lambda_{2}=-\lambda_{1}
$$

where $\alpha(t)$ is a $C^{\infty}$ function such that

$$
\left\{\begin{array}{l}
\alpha\left(\beta_{j}\right)=0, \quad(-1)^{j} \alpha(t)>0 \quad \text { if } \quad t \in\left(\beta_{j}, \beta_{j+1}\right) \\
\alpha^{\prime}\left(\beta_{j}\right) \neq 0, \quad \int_{\beta_{j}}^{\beta_{j+1}}|\alpha(t)| d t=2^{-(\jmath+1)}
\end{array}\right.
$$

for a sequence $\left\{\beta_{j}\right\}_{j=0}^{\infty}$ satisfying 


$$
0=\beta_{0}<\beta_{1}<\cdots<\beta_{j}<\cdots<T, \quad \beta_{j} \rightarrow T \quad(j \rightarrow \infty) .
$$

Here $T$ is a fixed positive. We can show the existence of $\alpha(t)$ in the same way as in Proposition 1.3 of [5].

If we take $\beta_{j}(j=1,2, \cdots)$ as a fixed positive $t_{0}$ in the definition of the trajectory, we have $2^{j}$ admissible trajectories issuing from $\left(0, \eta^{0}\right), \eta^{0} \neq 0$ whose end points are $\left( \pm(2 k-1) 2^{-j}, \eta^{0}\right), k=1, \cdots, 2^{j-1}$ (see Fig.) Each

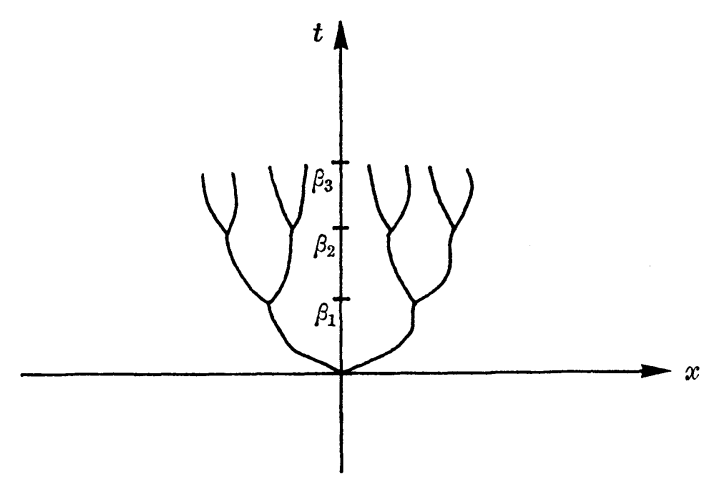

adm. traj. satisfies hypotheses of Theorem 1. In fact, (H-1) and (H-2) are verified because each adm. traj. is isolated in the sense of the Definition 1.2. We can take arbitrary positive $\sigma$ smaller than 1 in $(\mathrm{H}-3)$ because $\alpha^{\prime}\left(\beta_{k}\right) \neq 0(k<j)$. It is easy to check the hypothesis (H-2) because $M_{j}$ $(j=1,2)$ in (1.2) are identity and because

$$
\begin{aligned}
\partial_{t_{q}} d_{J_{\mu}^{\prime}}\left(\tilde{t}_{\mu}\right) & =\partial_{t_{q}} d_{j_{q}}\left(t_{q-1}, t_{q}\right)+\partial_{t_{q}} d_{j_{q+1}}\left(t_{q}, t_{q+1}\right) \\
& = \pm \alpha\left(t_{q}\right)-\left(\mp \alpha\left(t_{q}\right)\right)= \pm 2 \alpha\left(t_{q}\right) .
\end{aligned}
$$

It follows from Theorem 1 that there exists an initial value $G$ such that

$$
W F G=\left\{\left(0, c \eta^{0}\right) ; c>0\right\}
$$

$$
W F U\left(\beta_{j}\right)=\left\{\left( \pm(2 k-1) 2^{-j}, c \eta^{0}\right) ; k=1, \cdots, 2^{j-1}, c>0\right\},
$$

where $U$ is the solution of (C.P) for the initial value $G$. Here we used Theorem 3.4 of [9] to show another direction of inclusion in (4.3). Because we take $G$ uniformly with respect to $\beta_{j}(j=1,2, \cdots)$ and because $W F U$ $\subset T^{*}\left(R_{t}^{1} \times R_{x}^{1}\right)$ is closed, we have

THEOREM 2. Let $L$ be the hyperbolic system of this section. Then there exists an initial value $G$ such that

$$
W F G=\left\{\left(0, c \eta^{0}\right) ; c>0\right\} \quad W F U(T)=[-1,1] \times\left\{c \eta^{0} ; c>0\right\},
$$


where $U$ is the solution of (C.P).

Corollary. For $G$ and $U$ in Theorem 2 we have

$$
\left\{\begin{array}{l}
\text { sing supp } G=\{0\} \\
\text { sing supp } U(T)=[-1,1] .
\end{array}\right.
$$

As stated in Introduction, the result analogous to (4.4) had been given by [5] by using the work of [12], that is, the precise propagation of singularities for the equation

$$
\partial_{t}^{2}-t^{2 s} \partial_{x}^{2}-a t^{s-1} \partial_{x}
$$

where $a$ is a constant and $s=1,2, \cdots$, (cf. [1] [3]).

\section{REFERENCES}

[1] S. Alinhac, Branching of singularities for a class of hyperbolic operators, Indiana Univ. Math. J., 27 (1978), 1027-1037.

[2] J. J. Duistermaat, Fourier integral operators, Courant Institute of Mathematical Sciences, 1973.

[ 3 ] N. Hanges, Parametrices and propagation of singularities for operators with noninvolutive characteristics, Indiana Univ. Math. J., 28 (1979), 87-97.

[4] L. Hörmander, Spectral analysis of singularities, Seminar of Singularities of Solutions of Linear Partial Differential Equations, Princeton University Press 1979, $3-49$.

[5] W. Ichinose and H. Kumano-go, On the propagation of singularities with infinitely many branching points for a hyperbolic equation of second order, Comm. Partial Differential Equations, 6 (1981), 568-623.

[6] C. Iwasaki and Y. Morimoto, Propagation of singularities of solutions for a hyperbolic system with nilpotent characteristics, I, Comm. Partial Differential Equations, 7 (1982), 743-794.

[ 7 ] - Propagation of singularities of solutions for a hyperbolic system with nilpotent characteristics, II, Comm. Partial Differential Equations, 9 (1984), 14071436.

[ 8 ] H. Kumano-go, K. Taniguchi and Y. Tozaki, Multi-product of phase functions for Fourier integral operators with an application, Comm. Partial Differential Equations, 3 (1978), 349-380.

[9] H. Kumano-go and K. Taniguchi, Fourier integral operators of multi phase and the fundamental solution for a hyperbolic system, Funkcial Ekvac., 22 (1979), 161-196.

[10] Y. Morimoto, On the propagation of the wave front set of a solution for a hyperbolic system, Math. Japonica, 27 (1982), 501-508.

[11] - Propagation of wave front set in Gevrey class for an example of hyperbolic system, Nagoya Math. J., 101 (1986), 131-150.

[12] K. Taniguchi and Tozaki, A hyperbolic equation with double characteristics which has a solution with branching singularities, Math. Japonica, 25, (1980), 279-300.

[13] S. Wakabayashi, Singularities of solutions of the Cauchy problem for hyperbolic systems in Gevrey classes, Japan. J. Math., 11 (1985), 157-201. 
[14] - Singularities of solutions of the Cauchy problem for symmetric hyperbolic systems, Comm. Partial Differential Equations, 9 (1984), 1147-1177.

Chisato Iwasaki

Department of Mathematics

Osaka University

Toyonaka, Osaka 560, Japan

Yoshinori Morimoto

Department of Engineering

Mathematics, Nagoya University

Chikusa-ku, Nagoya 464, Japan 